\title{
Correction to: Computational Analysis of Structural Variation in Cancer Genomes
}

\section{Matthew Hayes}

\section{Correction to:}

Chapter 3 in: Alexander Krasnitz (ed.),

Cancer Bioinformatics, Methods in Molecular Biology, vol. 1878, https://doi.org/10.1007/978-1-4939-8868-6_3

The author originally had the wrong programming command and the chapter was inadvertently published with error. The same has been updated later as below

samtools view -b -F 14 alignment.sorted.nodup.bam \

$>$ alignment.sorted.nodup.nocon.bam 